\title{
The channelopathies: novel insights into molecular and genetic mechanisms of human disease
}

\author{
Robert S. Kass \\ Department of Pharmacology, Columbia University Medical Center, New York, New York, USA.
}

\begin{abstract}
Ion channels are pore-forming proteins that provide pathways for the controlled movement of ions into or out of cells. Ionic movement across cell membranes is critical for essential and physiological processes ranging from control of the strength and duration of the heartbeat to the regulation of insulin secretion in pancreatic $\beta$ cells. Diseases caused by mutations in genes that encode ion channel subunits or regulatory proteins are referred to as channelopathies. As might be expected based on the diverse roles of ion channels, channelopathies range from inherited cardiac arrhythmias, to muscle disorders, to forms of diabetes. This series of reviews examines the roles of ion channels in health and disease.
\end{abstract}

\section{Introduction}

Ion channels are a diverse group of pore-forming proteins that cross the lipid membrane of cells and selectively conduct ions across this barrier. Ion channels coordinate electrical signals in most tissues and are thus involved in every heartbeat, every movement, and every thought and perception. They have evolved to selectively provide pathways for ions to move down their electrochemical gradients across cell membranes and either depolarize cells, by moving positively charged ions in, or repolarize cells, by moving positively charged ions out.

During the past 50 years, our understanding of the roles and molecular structure of ion channels has grown at a rapid pace and has bridged fundamental basic research with advances in clinical medicine. The link between basic science and clinical medicine has been the discovery of human diseases linked to mutations in genes coding for ion channel subunits or proteins that regulate them: the channelopathies.

Ion channels: from squid giant axons to atomic structure Before 1982, insight into mechanisms underlying the ionic basis of electrical activity in excitable cells was limited to model systems. Our understanding of these mechanisms was based largely on the beautiful work of Hodgkin, Huxley, and Cole, among others, who unraveled the ionic basis of nerve excitation in the squid giant axon (1-4) and subsequently showed that similar mechanisms were responsible for excitation and contraction of amphibian skeletal muscle (5). Using mammalian preparations, other groups soon demonstrated similar, though more complex, mechanisms underlying electrical activity in the heart (6). However, the link between mechanisms in these model systems and human physiology remained indirect. This changed dramatically in 1982 when the first ion channel, acetylcholine receptor $\alpha$-subunit, was cloned $(7,8)$. Molecular biology provided the techniques to identify genes encoding ion channels, and, as a result, a plethora of channels has been discovered to be critical to the physiological function of vir-

Nonstandard abbreviations used: LQTS, long QT syndrome.

Conflict of interest: The author has declared that no conflict of interest exists.

Citation for this article: J. Clin. Invest. 115:1986-1989 (2005).

doi:10.1172/JCI26011. tually every tissue, controlling such diverse functions as hearing and insulin secretion. The combination of genetic identification of multiple channel genes and the development of patch-clamp electrophysiological procedures by Neher and Sakmann (9) made it possible to analyze in great detail the functional properties of ion channels in small cells, eliminating the restriction to model systems and extending understanding of the roles of molecular structures in the control of channel function.

The crystal structure of a bacterial potassium channel was solved in 1998 (10), revealing, at the atomic level, the structural basis of fundamental mechanisms of this class of ion channels. Insight into channel structure clarified the manner in which the channels open and close; the structural basis for selection of ions that can pass through the open channel pore; and the mechanism by which the channel proteins sense changes in transmembrane voltage that control the open or closed conformational states of the channel (Figure 1) (11). Thus investigations of ion channel proteins bridge fundamental physics with function of biologically critical proteins. But the link to human disease has come from clinical investigations of congenital disorders and the discoveries that defects in genes coding for ion channels or ion channel regulatory subunits cause diverse disease states. The number of diseases linked to these mutations is so large that the term "channelopathy" has been introduced to define this class of disease (Table 1).

In this issue of the JCI, we have assembled a series of review articles to provide the most up-to-date information on channelopathies and to assess how investigations of these disorders have increased our understanding of the mechanisms of human disease. Rather than serving as an extensive catalog of all channelopathies, these papers impart both a historical perspective and an opportunity to understand how mutations that cause common changes in ion channel function may be associated with diverse diseases depending on the tissues in which the channels are expressed.

\section{Sodium channels}

The channelopathies are introduced by A.L. George Jr. with a broad discussion of the impact of mutations of voltage-gated sodium channels on human disease (12). Mutations in genes coding for sodium channels are now known to cause nearly 20 disorders ranging from skeletal muscle defects to dysfunction of the nervous system (12). 
A

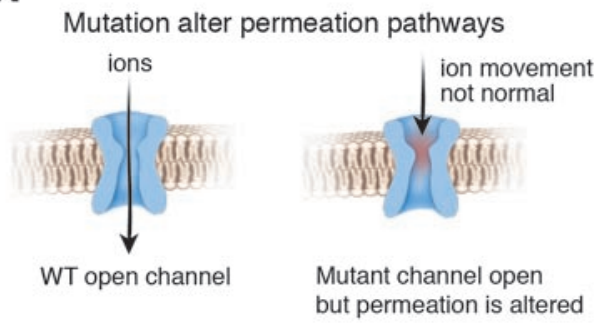

B Mutations change channel activation

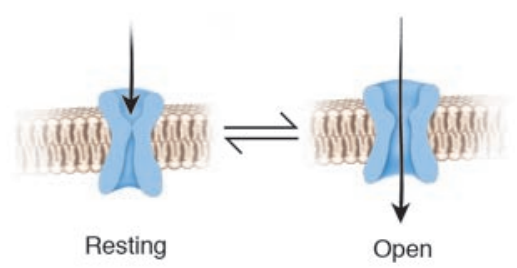

C Mutations change channel inactivation process

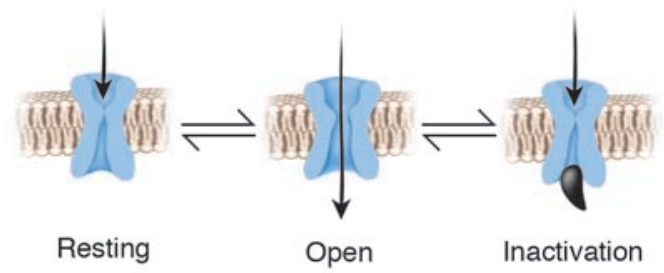

Investigation into the functional consequences of these mutations in heterologous expression systems has revealed novel mechanisms underlying disease processes (13-15), mutation-specific therapeutic approaches to disease management $(16,17)$, and, importantly, common functional mechanisms underlying such diverse diseases as cardiac arrhythmias and seizure disorders $(12,18)$.

Sodium channels open in response to membrane depolarization and, while open, provide the pathway for the inward movement of positively charged sodium ions. This results in a regenerative process that is necessary for conduction of electrical impulses in nerve and muscle. Within milliseconds of opening, most voltagegated sodium channels enter a nonconducting inactivated state. The rapid transition from open to inactivated states is necessary to control durations of electrical impulses in all tissues. Most inherited sodium channel mutations that are associated with human disease alter the inactivation process and hence alter the essential control of electrical-impulse duration that is effected by transitions into the inactivated state. Analysis of skeletal muscle disorders identified some of the first channelopathies associated with mutations that alter sodium channel inactivation. In this review series, Jurkat-Rott and Lehmann-Horn describe these and other skeletal muscle channelopathies (19). These authors provide an overview of the roles of sodium channels in muscle physiology and explain how mutations that alter channel inactivation may result in the clinical pathologies and myotonias. They note that in $\mathrm{Na}^{+}$channel myotonia and paramyotonia, mutation-induced gating defects of the $\mathrm{Na}^{+}$channels destabilize the inactivated state of

\section{Figure 1}

Inherited mutations alter ion channel function and structure and cause human disease. Mutations may alter the permeation pathway (A) to inhibit the movement of ions through an open channel pore and may also alter ion channel gating by changing either the process by which channels open (activate) (B) or the process by which they inactivate (C). Transitions from the open to the inactivated state reduce the number of channels that are available to conduct ions. Mutations that destabilize the inactivated, nonconducting state of the channel are gain-of-function mutations and are common to diverse diseases, including LQTS, certain forms of epilepsy, and muscle disorders such as hyperkalemic paralysis.

the channel such that inactivation may be slowed or incomplete. This results in hyperexcitability, repetitive muscle stimulation, and fatigue (19). A critical discussion of muscle channelopathies that may be caused by mutations in other ion channel genes, by antibodies directed against ion channel proteins, or by changes of cell homeostasis leading to aberrant splicing of ion channel RNA or to disturbances of modification and localization of channel proteins is also presented (19).

The importance of mutation-altered sodium channel inactivation to human disease is further explored by Meisler and Kearney (20), who present a detailed discussion of the roles of sodium channel mutations in epilepsy and other seizure disorders. They point out that since the first mutations of the neuronal sodium channel SCN1A were identified 5 years ago, more than 150 mutations have been described in patients with epilepsy, and they suggest that sodium channel mutations may be significant factors in the etiology of numerous neurological diseases and may contribute to psychiatric disorders as well. Interestingly, mutations related to epilepsy predominantly affect sodium channel inactivation. In these cases, defective inactivation of the channels could contribute to hyperexcitability. Thus similar channel mutations may have diverse and markedly different clinical consequences depending on the tissue in which the channel is expressed.

\section{Long QT syndrome}

Long QT syndrome (LQTS), a rare genetic disorder associated with life-threatening arrhythmias, is next reviewed briefly by Moss and Kass (21). LQTS investigations have provided fundamental new insight into the molecular and genetic basis of electrical signaling in the heart. To date, mutations in at least 8 different genes have been associated with LQTS. The most prevalent LQTS genes code for ion channel subunits, but regulatory proteins are also associated with this disorder. Though LQTS is caused by a diverse group of genes, the common clinical phenotype is delayed ventricular repolarization, which, in most cases, is caused by mutation-induced loss of potassium channel function or a gain of sodium channel function. The mutation-altered channel function that underlies LQTS is remarkably similar to altered channel function in epilepsy and skeletal muscle disorders, and this similarity provides insight into the physiologically essential balance of ion channel function in diverse cells and tissue.

An important contribution of LQTS investigations discussed by Moss and Kass has been the discovery not only of mutation-specific risk factors for different genetic variants of LQTS $(22,23)$ but of mutation-specific therapeutic strategies to manage the disorder $(16,24)$. Because the clinical phenotype, prolonged QT interval on the ECG, is determined by routine noninvasive procedures, LQTS 


\section{Table 1}

Selected channelopathies reviewed in this series

\begin{tabular}{|c|c|c|c|c|}
\hline Protein & Gene & Disease & Functional defect & Reference \\
\hline $\mathrm{Na}_{v} 1.1$ & SCN1A & Generalized epilepsy with febrile seizures plus (GEFS+) & Hyperexcitability & 12,20 \\
\hline $\mathrm{Na}_{v} 1.2$ & SCN2A & Generalized epilepsy with febrile and afebrile seizures & Hyperexcitability & 12,20 \\
\hline $\mathrm{Na}_{v} 1.4$ & SCN4A & $\begin{array}{c}\text { Paramyotonia congenita, potassium-aggravated myotonia, } \\
\text { hyperkalemic periodic paralysis }\end{array}$ & Hyperexcitability & $12,19,20$ \\
\hline $\mathrm{Na}_{v} 1.5$ & SCN5A & LQTS/Brugada syndrome & Heart action potential & 21 \\
\hline SCN1B & SCN1B & Generalized epilepsy with febrile seizures plus (GEFS+) & Hyperexcitability & 12,20 \\
\hline KCNQ1 & KCNQ1 & $\begin{array}{l}\text { Autosomal-dominant LQTS with deafness } \\
\text { Autosomal-recessive LQTS }\end{array}$ & $\begin{array}{c}\text { Heart action potential/inner ear } \mathrm{K}^{+} \text {secretion } \\
\text { Heart action potential }\end{array}$ & 21 \\
\hline KCNH2 & KCNH2 & LQTS & Heart action potential & 21 \\
\hline Kir2.1 & KCNJ2 & LQTS with dysmorphic features & Heart action potential & 21 \\
\hline HERG & $\mathrm{KCNH} 2$ & Congenital and acquired LQTS & $\begin{array}{l}\text { Heart action potential and excessive } \\
\text { responses to drugs }\end{array}$ & 21,26 \\
\hline Ankyrin-B & $A N K B$ & LQTS & Heart action potential & 21 \\
\hline $\mathrm{Ca}_{v} 1.2$ & CACNA2 & Timothy syndrome & Multisystem disorders & 21 \\
\hline Kir6.2 & KCNJ11 & $\begin{array}{c}\text { Persistent hyperinsulinemic hypoglycemia of infancy } \\
\text { Diabetes mellitus }\end{array}$ & $\begin{array}{l}\text { Insulin hypersecretion } \\
\text { Insulin hyposecretion }\end{array}$ & 29 \\
\hline SUR1 & SUR1 & Persistent hyperinsulinemic hypoglycemia of infancy & Insulin hyposecretion & 29 \\
\hline SUR2 & SUR2 & Dilated cardiomyopathy & Metabolic signaling & 29 \\
\hline KCNE1 & KCNE1 & $\begin{array}{l}\text { Autosomal-dominant LQTS with deafness } \\
\text { Autosomal-dominant LQTS }\end{array}$ & $\begin{array}{l}\text { Heart action potential } \\
\text { Heart action potential }\end{array}$ & 21 \\
\hline KCNE2 & KCNE2 & LQTS & Heart action potential & 21 \\
\hline CFTR & $A B C C 7$ & Cystic fibrosis & Epithelial transport defect & 28 \\
\hline CIC-1 & CLCN1 & Myotonia (autosomal-recessive or -dominant) & Defective muscle repolarization & 19,28 \\
\hline CIC-5 & CLCN5 & Dent disease & Defective endosome acidification & 28 \\
\hline CIC-7 & CLCN7 & Osteopetrosis (recessive or dominant) & Defective bone resorption & 28 \\
\hline $\mathrm{CIC}-\mathrm{Kb}$ & CLCNKB & Bartter syndrome type III & Renal salt loss & 28 \\
\hline RyR1 & $R y R 1$ & Central core disease, malignant hyperthermia & Abnormal muscle activity & 19,27 \\
\hline RyR2 & RyR2 & Catecholaminergic polymorphic tachycardia & Exercise-related cardiac arrhythmias & 27 \\
\hline
\end{tabular}

has led to fundamentally novel understanding of the processes that control and regulate electrical activity in the heart.

Drug-induced QT prolongation defines “acquired” LQTS (25). Though, by definition, this disorder is not due to mutationinduced QT prolongation due directly to changes in ion channel gene products, considerable evidence indicates that, in many cases, variations in ion channel genes that are not sufficiently severe to be classified as mutations, but instead are referred to as polymorphisms, may contribute to unique drug responses in carriers of these gene variants. Drug-induced QT prolongation, so-called acquired LQTS, is reviewed by Roden and Viswanathan (26).

\section{Ion channels in cellular organelles and nonexcitable cells}

Ion channels expressed in plasma membranes affect cellular excitability in nerve and muscle, but expression of ion channels in nonexcitable cells and membranes of intracellular organelles such as the sarcoplasmic reticulum, lysosomes, and mitochondria confers a broad range of functional roles to these important proteins. Mutations in channels responsible for the control of intracellular calcium release from the principal intracellular calcium store in muscle, the sarcoplasmic reticulum, have only recently been discovered to underlie a class of intracellular calcium-dependent arrhythmias and disorders. Priori and Napolitano review the current knowledge about the mutations of the gene encoding the cardiac ryanodine receptor $(R y R 2)$ that cause cardiac arrhythmias (27). These authors discuss the similarities between the mutations identified in genes coding for calcium release channels (ryanodine receptors). In the heart, mutations in the ryanodine receptor $R y R 2$ cause cardiac arrhythmias. In skeletal muscle, mutations in the ryanodine receptor RyR1 cause malignant hyperthermia and central core disease.

Finally, reviews by Jentsch et al. (28) and Ashcroft (29) concentrate on channelopathies in classically nonexcitable tissue. Jentsch et al. (28) focus on diseases related to transepithelial transport and on disorders involving vesicular $\mathrm{Cl}^{-}$channels. As is pointed out in their review, the transport of anions across cellular membranes is crucial for multiple physiological functions that include the control of electrical excitability of muscle and nerve, transport of salt and water across epithelia, and the regulation of cell volume or the acidification and ionic homeostasis of intracellular organelles. Consequently, mutations in $\mathrm{Cl}^{-}$channels are associated with diverse human disease.

ATP-sensitive potassium $\left(\mathrm{K}_{\mathrm{ATP}}\right)$ channels, so named because they are inhibited by intracellular ATP, couple cell metabolism to electrical activity of the plasma membrane. When intracellular ATP concentrations fall, these channels open and hyperpolarize cells. Conversely, when intracellular ATP concentrations are elevated, $\mathrm{K}_{\mathrm{ATP}}$ channels close, resulting in membrane depolarization. In pancreatic $\beta$ cells, membrane depolarization induced by closure of these channels results in activation of voltage-dependent calcium channels and, consequently, glucose-dependent insulin secretion. Thus, $\mathrm{K}_{\mathrm{ATP}}$ channels serve as targets for drugs that are used to treat and control type 2 diabetes, such as the sulphonylureas. The review by Ashcroft (29) highlights insulin secretory disorders, 
such as congenital hyperinsulinemia and neonatal diabetes, that result from mutations in $\mathrm{K}_{\mathrm{ATP}}$ channel genes and considers whether defective regulation of $\mathrm{K}_{\mathrm{ATP}}$ channel activity contributes to the etiology of type 2 diabetes.

\section{Summary}

The aim of this special JCI series on channelopathies is to review selected disorders caused by mutations that result in defective ion channel function, regulation, or expression. Together, this collection of articles illustrates the impact that investigation of these diseases has had on our understanding of the mechanistic basis of human disease, revealing critical roles of ion channel function in physiological processes as diverse as muscle activation and regula-

1. Hodgkin, A.L., and Huxley, A.F. 1952. A quantitative description of membrane current and its application to conduction and excitation in nerve. J. Physiol. 117:500-544.

2. Hodgkin, A.L., and Rushton, W.A.H. 1946. The electrical constants of a crustacean nerve fiber. Proc. R. Soc. Lond. B. B133:444-479.

3. Hodgkin, A.L., and Huxley, A.F. 1952. Propagation of electrical signals along giant nerve fibers. Proc. $R$. Soc. Lond. B Biol. Sci. 140:177-183.

4. Cole, K.S. 1979. Mostly membranes (Kenneth S. Cole). Annu. Rev. Physiol. 41:1-24.

5. Hodgkin, A.L., and Horowicz, P. 1959. Movements of $\mathrm{Na}$ and $\mathrm{K}$ in single muscle fibres. J. Physiol. 145:405-432.

6. Weidmann, S. 1952. The electrical constants of Purkinje fibres. J. Physiol. 118:348-360.

7. Noda, M., et al. 1982. Primary structure of alphasubunit precursor of Torpedo californica acetylcholine receptor deduced from cDNA sequence. Nature. 299:793-797.

8. Giraudat, J., Devillers-Thiery, A., Auffray, C., Rougeon, F., and Changeux, J.P. 1982. Identification of a cDNA clone coding for the acetylcholine binding subunit of Torpedo marmorata acetylcholine receptor. EMBO J. 1:713-717.

9. Neher, E., and Sakmann, B. 1976. Single-channel currents recorded from membrane of denervated frog muscle fibres. Nature. 260:799-802.

10. Doyle, D.A., et al. 1998. The structure of the potassium channel: molecular basis of $\mathrm{K}+$ conduction and selectivity. Science. 280:69-77.

11. MacKinnon, R. 2003. Potassium channels. FEBS

tion of insulin secretion. Remarkable conservation of the essential function of ion channels emerges from this series of review articles in this issue of the JCI - function that, when disrupted in neurons, may cause epilepsy, but, when disrupted in the heart, causes fatal cardiac arrhythmias. The lessons from these studies are dramatic and clearly call for additional collaborative investigation in which clinical and preclinical scientists, working together, continue to unravel the molecular basis of diverse human disorders.

Address correspondence to: Robert S. Kass, Department of Pharmacology, Columbia University Medical Center, 630 West 168th Street, PH 7W318, New York, New York 10032, USA. Phone: (212) 305-7444; Fax: (212) 305-8780; E-mail: rsk20@columbia.edu.

Lett. 555:62-65.

12. George, A.L., Jr. 2005. Inherited disorders of voltage-gated sodium channels. J. Clin. Invest. 115:1990-1999. doi:10.1172/JCI25505.

13. Clancy, C.E., Tateyama, M., Liu, H., Wehrens, X.H., and Kass, R.S. 2003. Non-equilibrium gating in cardiac $\mathrm{Na}+$ channels: an original mechanism of arrhythmia. Circulation. 107:2233-2237.

14. Clancy, C.E., and Kass, R.S. 2005. Inherited and acquired vulnerability to ventricular arrhythmias: cardiac $\mathrm{Na}^{+}$and $\mathrm{K}+$ channels. Physiol. Rev. 85:33-47.

15. Nuyens, D., et al. 2001. Abrupt rate accelerations or premature beats cause life-threatening arrhythmias in mice with long-QT3 syndrome. Nat. Med. 7:1021-1027.

16. Benhorin, J., et al. 2000. Effects of flecainide in patients with new SCN5A mutation: mutationspecific therapy for long-QT syndrome? Circulation. 101:1698-1706.

17. Abriel, H., Wehrens, X.H., Benhorin, J., Kerem, B., and Kass, R.S. 2000. Molecular pharmacology of the sodium channel mutation D1790G linked to the long-QT syndrome. Circulation. 102:921-925.

18. Lossin, C., Wang, D.W., Rhodes, T.H., Vanoye, C.G., and George, A.L., Jr. 2002. Molecular basis of an inherited epilepsy. Neuron. 34:877-884.

19. Jurkat-Rott, K., and Lehmann-Horn, F. 2005. Muscle channelopathies and critical points in functional and genetic studies. J. Clin. Invest. 115:2000-2009. doi:10.1172/JCI25525.

20. Meisler, M.H., and Kearney, J.A. 2005. Sodium channel mutations in epilepsy and other neurological disorders. J. Clin. Invest. 115:2010-2017.
doi:10.1172/JCI25466.

21. Moss, A.J., and Kass, R.S. 2005. Long QT syndrome: from channels to cardiac arrhythmias. J. Clin. Invest. 115:2018-2024. doi:10.1172/JCI25537.

22. Moss, A.J., et al. 2002. Increased risk of arrhythmic events in long-QT syndrome with mutations in the pore region of the human ether-a-go-go-related gene potassium channel. Circulation. 105:794-799.

23. Schwartz, P.J., et al. 2001. Genotype-phenotype correlation in the long-QT syndrome: gene-specific triggers for life-threatening arrhythmias. Circulation. 103:89-95.

24. Moss, A.J., et al. 2000. Effectiveness and limitations of beta-blocker therapy in congenital long-QT syndrome. Circulation. 101:616-623.

25. Fenichel, R.R., et al. 2004. Drug-induced torsades de pointes and implications for drug development. J. Cardiovasc. Electrophysiol. 15:475-495.

26. Roden, D.M., and Viswanathan, P.C. 2005. Genetics of acquired long QT syndrome. J. Clin. Invest. 115:2025-2032. doi:10.1172/JCI25539.

27. Priori, S.G., and Napolitano, C. 2005. Cardiac and skeletal muscle disorders caused by mutations in the intracellular $\mathrm{Ca}^{2+}$ release channels. J. Clin. Invest. 115:2033-2038. doi:10.1172/JCI25664.

28. Jentsch, T.J., Maritzen, T., and Zdebik, A.A. 2005. Chloride channel diseases resulting from impaired transepithelial transport or vesicular function. J. Clin. Invest. 115:2039-2046. doi:10.1172/ JCI25470.

29. Ashcroft, F.M. 2005. ATP-sensitive potassium channelopathies: focus on insulin secretion. J. Clin. Invest. 115:2047-2058. doi:10.1172/JCI25495. 